\title{
Assessing the Effect of SNPs on Litter Traits in Pigs
}

\author{
Lyubov V. Getmantseva $\mathbb{D}^{1},{ }^{1}$ Siroj Yu Bakoev, ${ }^{1}$ Varvara S. Shevtsova, ${ }^{2}$ Anatoly Yu Kolosov, ${ }^{1,3}$ \\ Neckruz F. Bakoev, ${ }^{1}$ and Maria A. Kolosova ${ }^{2,3}$ \\ ${ }^{1}$ Federal Science Center for Animal Husbandry named after Academy Member L.K. Ernst, Dubrovitsy 142132, Russia \\ ${ }^{2}$ Southern Federal University, Rostov-on-Don 344006, Russia \\ ${ }^{3}$ Don State Agrarian University, Persianovski 346493, Russia
}

Correspondence should be addressed to Lyubov V. Getmantseva; ilonaluba1@mail.ru

Received 31 October 2019; Revised 4 June 2020; Accepted 15 June 2020; Published 30 July 2020

Academic Editor: Devapriya Choudhury

Copyright (C) 2020 Lyubov V. Getmantseva et al. This is an open access article distributed under the Creative Commons Attribution License, which permits unrestricted use, distribution, and reproduction in any medium, provided the original work is properly cited.

\begin{abstract}
The reproductive ability of sows is the principle of continuous and efficient production, based on such traits as the number of piglets, the total number of parities, and the period of economic use. Currently, SNPs associated with the TNB and NBA are presented in the PigQTLdb. The aim of this work was the assessment of the SNP effects on the litter traits in Large White (LW, $n=502$ ) and Landrace (LN, $n=432$ ) sow breeds in a farm in Russia. 9 SNPs (SNP_1: rs80956812; SNP_2: rs81471381; SNP_3: rs80891106; SNP_4: rs81399474; SNP_5: rs81421148; SNP_6: rs81242222; SNP_7: rs81319839; SNP_8: rs81312912; SNP_9: rs80962240) were selected for the study. Associative analysis was performed using the GLM procedure in R version 3.5.1. The analysis of reproductive traits was carried out according to the results of the first parity, the second and subsequent parities, and totals for lifetime of sows. The significant effect on litter traits in LW was determined for SNP rs80956812, SNP rs81471381, SNP rs81421148, and SNP rs81399474. The significant effect on litter traits in LN was determined for SNP rs81421148 and SNP rs81319839. AKT3 gene was identified as perspective candidate gene, whose biological functions, as well as the results obtained in our work and in other studies, indicate its potential role in the reproductive process regulation in pigs. In general, the data obtained help to explain the genetic mechanisms of reproductive traits.
\end{abstract}

\section{Introduction}

Selection of specific individuals with desirable traits is fundamental for animal breeding. The scope and complexity of selection, as well as the number and size of populations, in traditional breeding programs require new tools based on recent advances in molecular biology and genetics. Therefore, the interest of scientists is focused on the study of the molecular genetic basis of the farm animal productive traits. Nowadays, genome-wide association study (GWAS) is the most powerful tool for studying polygenic traits genetic architecture [1, 2]. SNP genotyping panels are the most affordable solution for GWAS. These panels have been developed to include high (HD), medium (MD), or low (LD) density distribution of markers across the genome. Despite certain deficiencies [3], SNP panels have gained great popularity for the studies of quantitative trait genetic architecture in farm animals and pigs in particular [4-7]. One of the difficult tasks in pig breeding is improving the litter traits. The reproductive ability of sows is the principle of continuous and efficient production, based on such reproductive traits as the number of piglets, the total number of parities, and the period of economic use. The total number born (TNB) and the total number born alive (NBA) are the main characteristics of sows' litter traits [7-9]. These characteristics reflect the level of all physiological processes associated with fertilization, intrauterine development of fetuses, and birthing activity of sows and are easy to account for. In the last decades, the best linear unbiased prediction (BLUP) method has made a significant contribution to the improvement of the reproductive performance. However, the low heritability of reproductive traits (around 0.10) and their phenotypic manifestation limited by the sex of animals result in the need for developing new approaches to reveal 
the biological nature of reproductive abilities [10-12]. GWAS seems to be very useful for reproductive traits of genetic architecture studies. Currently, on the basis of genome map association results, 314 SNPs associated with TNB (225 SNPs) and NBA (89 SNPs) are presented in the PigQTLdb database [13]. The data obtained by GWAS allowed the identification of SNPs associated with litter traits in pigs. However, SNPs with signs of productivity may vary in different populations and also have a number of features due to the breed of pigs. For a better understanding of the SNP effects, it is necessary to conduct studies allowing the assessment of the repeatability of the obtained associations for different groups of pigs.

The aim of this work was to assess the effect of SNPs on litter traits in LW and LN pigs in a Russian breeding farm. To achieve this goal, it was necessary to select SNPs associated with TNB and NBA from the PigQTLdb and to develop techniques identifying these SNPs. Another goal was to identify prospective candidate genes to improve the reproductive qualities of pigs.

\section{Methods}

The research was carried out on the basis of the Center for Collective Use of Scientific Equipment "Bioresources and Bioengineering of Agricultural Animals" of the L.K. Ernst Federal Research Center for Animal Husbandry (https:// www.vij.ru/infrastruktura/ckp). Samples from the Unique Scientific Installation (UNU) "Bank of Genetic Materials of Animals and Birds" (https://www.vij.ru/infrastruktura/46infrastruktura/286-unikalnaya-nauchnaya-ustanovka-unu) were used for the work. For this collection, samples (tissue samples from the ear) were given by the owners of the breeding farms (according to their will). All methods were performed in accordance with the guidelines approved by the L.K. Ernst Federal Research Center for Animal Husbandry, Russia.

SNPs presented in the works of Sell-Kubiak et al. [14]; Bergfelder-Drüing et al. [15]; He et al. [16]; and Wang et al. [17] have been chosen for the study (Table 1). The names of these SNPs in accordance with the chips for genotyping on the basis of Ensembl Release 96 (April 2019) are presented in Add. File 1. The primers were designed based on available genomic sequences presented in the National Center for Biotechnology Information (NCBI) database (plus/minus $300 \mathrm{bp}$ from the certain SNP). Oligonucleotide primers and restriction endonucleases for polymerase chain reactionrestriction fragment length polymorphism (PCR-RFLP) identification of SNP were selected using the software Primer-BLAST (https://www.ncbi.nlm.nih.gov/tools/ primer-blast/) and NEBcutter V2.0 (http://nc2.neb.com/ NEBcutter2/index.php). In case of the restriction site absence, the pyrosequencing (PSQ) method was used. Selected primers, annealing temperature, and restriction endonuclease for PCR-RFLP are presented in Table 2.

The studies were carried out on LW $(n=502)$ and LN $(n=432)$ sow breeds in a farm in Russia. All the animals had the same conditions of housing and feeding. Genomic DNA was extracted from porcine tissue samples (ear aperture) using the DNA kit Extran-2 (Syntol, Russia) according to the manufacturer's instructions. The quantity, quality, and integrity of the DNA were assessed by Qubit 2.0 fluorimeter (Invitrogen/Life Technologies, USA) and a NanoDrop 8000 spectrophotometer (Thermo Fisher Scientific, USA).

The analysis of sows reproductive abilities was carried out according to the results of the first parity: total number born (TNB_1), total number born alive (NBA_1), litter weight of piglets born alive (BALWT_1); the results of the second and subsequent parities: total number born (TNB), total number born alive (NBA), litter weight of piglets born alive (BALWT); and totals for lifetime of sows: total number born (TNB_All), total number born alive (NBA_All), litter weight of piglets born alive (BALWT_All), and all parities (P_All). In accounting for P_All, the sows with at least three parities were selected.

\section{Statistical Analysis}

Associative analysis was performed using the GLM procedure in $\mathrm{R}$ version 3.5.1. The mixed model with the fixed effects was as follows: $\mathbf{Y i j}=\boldsymbol{\mu}+\mathbf{G i}+\mathbf{B j}+\mathbf{e j i}$, where $\mathbf{Y} \mathbf{i j}$ is the ijth trait observation value; $\boldsymbol{\mu}$ is the mean; $\mathbf{G i}$ is the effect of the ith genotypes; $\mathbf{B} \mathbf{j}$ is the effect of jth breeding; and eji is the random residual that corresponds to the trait observation value. To determine if the SNP had a significant additive effect, dominance effect, or both, contrasts for additive and dominance effects were tested for the most significant SNP. Additive effects were declared when the contrast between the effects of the two homozygous genotypes was significantly $(P \leq 0.01$ and $P \leq 0.05)$ or suggestively different $(P \leq 0.15)$. Dominance effects were declared when the contrast between the average effect of the two homozygous genotypes (AA and $\mathrm{BB})$ and the effect of the heterozygous genotype was significantly $(P \leq 0.01$ and $P \leq 0.05)$ or suggestively different $(P \leq 0.15)$. To adjust $P$ values, the Bonferroni method was used.

Additive and dominant SNP effects were calculated using the following formulas [18]: $\mathrm{a}=(\mathrm{BB}-\mathrm{AA}) / 2$ and $d=\mathrm{AB}-(\mathrm{BB}+\mathrm{AA}) / 2$, where $a$ is the additive effect; $d$ is the dominant effect; and $\mathrm{AA}, \mathrm{AB}$, and $\mathrm{BB}$ are the genotypes of SNP.

\section{Results}

The frequencies of alleles and genotypes of the studied SNP are presented in Table 3. LW pigs, in contrast to LN, were monomorphic on SNP_6. At the same time, polymorphism for SNP_1 in pigs LN was not detected. In addition, large differences in SNP_2, SNP_3, and SNP_7 between LW and LN should be noted. Further associative analysis was performed separately for the sows of LW and LN.

SNPs with the minor allele frequencies lower than 0.05 were excluded from the analysis. In LW, SNP_6 and SNP_8 were excluded; in LN, SNP_1 was excluded. The significant effect on litter traits in LW was determined for SNP_1 and SNP 2 on BALWT and for SNP_4 on BALWT_1 (Table 4). The suggestive effect on litter traits in LW was defined for SNP_3 on TNB_1, NBA_1, and P_All; for SNP_5 on TNB, 
TABLE 1: Selected SNPs for the study.

\begin{tabular}{|c|c|c|c|c|c|c|}
\hline \multirow{2}{*}{ Number of SNPs (in text) } & \multirow{2}{*}{ SNP } & \multirow{2}{*}{ SSC } & \multicolumn{2}{|c|}{ Location } & \multirow{2}{*}{ Genes } & \multirow{2}{*}{ Reference (breed) } \\
\hline & & & Sscrofa 11.1 & Sscrofa 10.2 & & \\
\hline SNP_1 & rs80956812 & 1 & 164674664 & 182418248 & SMAD6 & {$[14]$} \\
\hline SNP_2 & rs81471381 & 18 & 53672799 & 58861041 & SUGCT & {$[14]$} \\
\hline SNP_3 & rs80891106 & 7 & 73467314 & 78538720 & - & {$[16]$} \\
\hline SNP_4 & rs81399474 & 8 & 32370687 & 33985796 & - & {$[16]$} \\
\hline SNP_5 & rs81421148 & 10 & 16506621 & 18203672 & AKT3 & {$[15]$} \\
\hline SNP_6 & rs81242222 & 11 & 67129570 & 74240078 & - & {$[15]$} \\
\hline SNP_7 & rs81319839 & 4 & 18194352 & 19239772 & - & {$[17]$} \\
\hline SNP_8 & rs81312912 & 4 & 18196598 & 19237526 & & {$[17]$} \\
\hline SNP_9 & rs80962240 & 13 & 52784022 & 58478836 & FOXP1 & {$[17]$} \\
\hline
\end{tabular}

TABLE 2: Oligonucleotide primers and restriction endonucleases to identify the SNPs under study.

\begin{tabular}{|c|c|c|c|c|c|c|}
\hline $\begin{array}{l}\text { Number } \\
\text { of SNPs }\end{array}$ & SNP & Oligonucleotide primers & $\begin{array}{l}\text { Annealing } \\
\text { temperature }\end{array}$ & $\begin{array}{l}\text { Length of } \\
\text { amplicon } \\
\text { (bp) }\end{array}$ & $\begin{array}{c}\text { Restriction } \\
\text { endonuclease/ } \\
\text { PSQ }\end{array}$ & $\begin{array}{l}\text { Length of } \\
\text { restriction } \\
\text { fragments } \\
\quad(\mathrm{bp})\end{array}$ \\
\hline SNP_1 & rs80956812 & $\begin{array}{c}\text { F5' }^{\prime} \text {-GGCGCTAAGTGAGCTCTTG-3' } \\
\text { R55'-CCCTCACTGATGCAACTCTAAA-3' }\end{array}$ & & 296 & Bme18 I & 202, 94 \\
\hline SNP_2 & rs81471381 & $\begin{array}{l}\text { F5'-CCACGCTCTCTACAAGCCAA-3' } \\
\text { R5'-CCCCATTCACGGTCTTGGAA-3' }\end{array}$ & & 540 & SfaN I & 368,172 \\
\hline SNP_3 & rs80891106 & $\begin{array}{c}\text { F5 }^{\prime} \text {-TGACAAGCTTCAGACAGTTCCT-3' } \\
\text { R5'-TGCACTGAACCTTCACACACA-3' }\end{array}$ & & 332 & PSQ & \\
\hline SNP_4 & rs81399474 & $\begin{array}{c}\text { F5'-AGAACGAGGCTTCTTCCTGTT-3' } \\
\text { R5'-ACAGTCTAAAGCCTGATTTCCCT-3' }\end{array}$ & & 297 & PSQ & \\
\hline SNP_5 & rs81421148 & $\begin{array}{l}\text { F5'-TTCTGTACTTCTCCATCACAAGAA-3' } \\
\text { R5'-CGAAGACTTGTTTACGCATCATAG-3' }\end{array}$ & & 383 & Mnl I & 276,107 \\
\hline SNP_6 & rs81242222 & $\begin{array}{c}\text { F5'-TGCAGAGATTCCAGCAAGCC-3' } \\
\text { R5'-CATCTGGTTGGTTTGGTCGTG-3' }\end{array}$ & & 290 & PSQ & \\
\hline SNP_7 & rs81319839 & $\begin{array}{c}\text { F5'-GAAGCACCCAATGGGACTCT-3' } \\
\text { R5 }^{\prime} \text {-ATGAGGTTGTCTTGGCACCAT-3' }\end{array}$ & & 356 & BstMB I & 276,80 \\
\hline SNP_8 & rs81312912 & $\begin{array}{c}\text { F5 }^{\prime} \text {-ACAGGACAGTATGAAAAATCTGTTG-3' } \\
\text { R5'-GCTTCCCCCAGAAAGGACTG-3 }{ }^{\prime}\end{array}$ & & 302 & BstAU I & 241,61 \\
\hline SNP_9 & rs80962240 & $\begin{array}{l}\text { F5'-ATGGAGGAACCGGCTATGTG-3' } \\
\text { R5'-GCAGTCCTGCCCATGAGTAT-3' }\end{array}$ & & 461 & Vsp I & 350,111 \\
\hline
\end{tabular}

NBA_1, BALWT_1, and BALWT; for SNP_7 on TNB_1; and for SNP_9 on BALWT.

The significant effects of SNP_5 on NBA and BALWT and of SNP_7 on NBA_1 and BALWT_1 in LN have been revealed (Table 5). The suggestive effect of SNP_2 on TNB_All and NBA_All, of SNP_5 on TNB_1 and TNB, and of SNP_7 on TNB_1 and BALWT in LN has been identified.

The results of additive and dominant effects of all studied SNPs are presented in Add. File 2.

4.1. Additive and Dominant SNP Effects Specific to LW Sow. In general, the additive and dominant effects on litter traits in LW sow are determined for SNP_1, SNP_2, SNP_3, and SNP_5. The significant effect of the SNP_1 genotypes on BALWT is determined. The BALWT in the sows with the genotype SNP_1_BB was higher by $1.8 \mathrm{~kg}(P \leq 0.05)$ and $1.1 \mathrm{~kg}(P \leq 0.05)$ compared to the sows with the AA and AB genotypes, respectively. The negative dominant effect of
SNP_2 on TNB and BALWT has been found. In the sows with the genotype SNP_2_AB, compared to homozygous sows, TNB and BALWT there were lesser by $0.7(P \leq 0.05)$ and $1.0(P \leq 0.05)$, respectively. In assessing the SNP_3 genotypes, additive effects on litter traits in first parity and the dominant effect on $\mathrm{P} \_$All were revealed. The sows with the genotype SNP_3_BB had 3.1 more TNB_1 and NBA_1 $(P \leq 0.05)$ compared to the sows of the genotype SNP_3_AA. For the entire productive period, sows with the genotype SNP_3_AB had 0.5 more P_All than sows with homozygous genotypes. According to the results of parities, the suggestive dominant effect on TNB_All, NBA_All, and BALWT_All is observed during the entire period of keeping. Analysis of the SNP_5 genotypes showed dominant effects on NBA_1 and BALWT_1, as well as additive effects on TNB, NBA, and BALWT. In the sows with the genotype SNP_5_AB, NBA_1 was smaller by $1.0(P \leq 0.05)$ and BALWT_1 was smaller by 1.6 $(P \leq 0.05)$ than these traits in the sows with homozygous 
TABLE 3: Frequency of alleles and genotypes.

\begin{tabular}{|c|c|c|c|c|c|c|c|c|}
\hline \multirow{3}{*}{ SNP } & \multicolumn{6}{|c|}{ Genotype } & \multicolumn{2}{|c|}{ Allele } \\
\hline & \multicolumn{2}{|c|}{$\mathrm{AA}$} & \multicolumn{2}{|c|}{$\mathrm{AB}$} & \multicolumn{2}{|c|}{$\mathrm{BB}$} & \multirow{2}{*}{ A } & \multirow{2}{*}{ B } \\
\hline & $n$ & $\%$ & $n$ & $\%$ & $n$ & $\%$ & & \\
\hline \multicolumn{9}{|c|}{ LW $(n=502)$} \\
\hline SNP_1 & 25 & 4.98 & 216 & 43.03 & 261 & 51.99 & 0.26 & 0.74 \\
\hline SNP_2 & 80 & 15.94 & 201 & 40.04 & 221 & 44.02 & 0.36 & 0.64 \\
\hline SNP_3 & 10 & 1.99 & 141 & 28.09 & 351 & 69.92 & 0.16 & 0.84 \\
\hline SNP_4 & 15 & 2.99 & 196 & 39.04 & 291 & 57.97 & 0.23 & 0.77 \\
\hline SNP_5 & 105 & 20.92 & 263 & 52.39 & 134 & 26.69 & 0.47 & 0.53 \\
\hline SNP_6 & 0 & 0.00 & 0 & 0.00 & 502 & 100.00 & 0.00 & 1.00 \\
\hline SNP_7 & 402 & 80.08 & 95 & 18.92 & 5 & 1.00 & 0.90 & 0.10 \\
\hline SNP_8 & 462 & 92.03 & 35 & 6.97 & 5 & 1.00 & 0.96 & 0.04 \\
\hline SNP_9 & 151 & 30.08 & 276 & 54.98 & 75 & 14.94 & 0.58 & 0.42 \\
\hline \multicolumn{9}{|c|}{$\mathrm{LN}(n=432)$} \\
\hline & AA & & $\mathrm{AB}$ & & & $\mathrm{BB}$ & A & B \\
\hline SNP_1 & 0 & 0.00 & 0 & 0.00 & 432 & 100.00 & 0.00 & 1.00 \\
\hline SNP_2 & 207 & 47.92 & 203 & 46.99 & 22 & 5.09 & 0.71 & 0.29 \\
\hline SNP_3 & 211 & 48.84 & 178 & 41.20 & 43 & 9.95 & 0.69 & 0.31 \\
\hline SNP_4 & 34 & 7.87 & 199 & 46.06 & 199 & 46.06 & 0.31 & 0.69 \\
\hline SNP _5 & 99 & 22.92 & 229 & 53.01 & 104 & 24.07 & 0.49 & 0.51 \\
\hline SNP_6 & 9 & 2.08 & 147 & 34.03 & 276 & 63.89 & 0.19 & 0.81 \\
\hline SNP_7 & 0 & 0.00 & 60 & 13.89 & 372 & 86.11 & 0.07 & 0.93 \\
\hline SNP_8 & 216 & 50.00 & 186 & 43.06 & 30 & 6.94 & 0.72 & 0.28 \\
\hline SNP_9 & 268 & 62.04 & 112 & 25.93 & 52 & 12.04 & 0.75 & 0.25 \\
\hline
\end{tabular}

TABLE 4: The SNP effects on the litter traits in LW.

\begin{tabular}{|c|c|c|c|c|c|c|c|}
\hline Traits & SNP_1 & SNP_2 & SNP_3 & SNP_4 & SNP_5 & SNP_7 & SNP_9 \\
\hline TNB_1 & 0.665 & 0.616 & 0.122 & 0.239 & 0.282 & 0.062 & 0.204 \\
\hline TNB & 0.976 & 0.189 & 0.710 & 0.383 & 0.100 & 0.686 & 0.730 \\
\hline TNB_All & 0.902 & 0.762 & 0.251 & 0.911 & 0.725 & 0.561 & 0.755 \\
\hline NBA_1 & 0.662 & 0.636 & 0.108 & 0.181 & 0.082 & 0.312 & 0.214 \\
\hline NBA & 0.765 & 0.202 & 0.745 & 0.264 & 0.149 & 0.837 & 0.733 \\
\hline NBA_All & 0.993 & 0.936 & 0.306 & 0.840 & 0.820 & 0.501 & 0.749 \\
\hline BALWT_1 & 0.620 & 0.304 & 0.191 & $0.033^{*}$ & 0.060 & 0.562 & 0.551 \\
\hline BALWT & $0.034^{*}$ & $0.046^{*}$ & 0.830 & 0.434 & 0.100 & 0.976 & 0.074 \\
\hline BALWT_All & 0.685 & 0.695 & 0.190 & 0.825 & 0.743 & 0.486 & 0.992 \\
\hline P_All & 0.933 & 0.968 & 0.129 & 0.683 & 0.738 & 0.425 & 0.537 \\
\hline
\end{tabular}

${ }^{*} P<0.05$. SNP_1: rs80956812; SNP_2: rs81471381; SNP_3: rs80891106; SNP_4: rs81399474; SNP_5: rs81421148; SNP_7: rs81319839; SNP_9: rs80962240. TNB_1: total number born of the first parity; TNB: total number born of the second and subsequent parities; TNB_All: total number born of lifetime sow; NBA_1: total number born alive of the first parity; NBA: total number born alive of the second and subsequent parities; NBA_All: total number born alive of lifetime sow; BALWT_1: litter weight of piglets born alive of the first parity; BALWT: litter weight of piglets born alive of the second and subsequent parities; BALWT_All: litter weight of piglets born alive of lifetime sow; P_All: all parities of lifetime sow.

genotype. Sows with the genotype SNP_5_BB had 1.1 more TNB $(P \leq 0.1), 0.9$ more NBA $(P \leq 0.05)$, and 1.3 more BALWT $(P \leq 0.05)$.

4.2. Additive and Dominant SNP Effects Specific to LN Sow. We have determined the additive and dominant effects of SNP_2, SNP_5, and SNP_7 on litter traits of LN sows. The analysis of SNP_2 genotypes showed significant dominant effects on TNB_All and NBA_All and suggestive dominant effects on BALWT_All and P_All. Sows with genotype SNP_2_AB had 8.9 more TNB_All $(P \leq 0.05), 7.4$ more NBA_All $(P \leq 0.05)$, and 0.5 more BALWT_All $(P \leq 0.1)$ and P_All $(P \leq 0.1)$ compared to homozygous sows. The additive effects of the genotypes SNP_5 were found for TNB, NBA, and BALWT. The sows with the genotype SNP_5_AA had 1.0 more TNB $(P \leq 0.05), 1.3$ more NBA $(P<0.01)$, and 1.8 more BALWT $(P<0.01)$, compared to sows with the genotype SNP_5_BB. An investigation of the first parity showed significant effects of the SNP_7 genotypes on NBA_1 and BALWT_1. The sows with the genotype SNP_7_AB had 1.8 more NBA_1 than $(P<0.01), 1.1$ more BALWT_1 $(P<0.01)$, and 1.4 more TNB_1 $(P \leq 0.1)$ compared to sows with the SNP_7_BB genotype. Significant effects of SNP_7 on subsequent parities were not observed statistically.

\section{Discussion}

Large White and Landrace breeds belong to the parent breeds used in three-breed breeding system of pigs at the first stage to obtain crossbreeding sows F1. Although the 
TABLE 5: The SNP effects on the litter traits in LN.

\begin{tabular}{lcccccccc}
\hline & SNP_2 & SNP_3 & SNP_4 & SNP_5 & SNP_6 & SNP_7 & SNP_8 & SNP_9 \\
\hline TNB_1 & 0.193 & 0.446 & 0.661 & 0.137 & 0.716 & 0.067 & 0.315 \\
TNB & 0.325 & 0.299 & 0.533 & 0.079 & 0.985 & 0.638 & 0.367 \\
TNB_All & 0.087 & 0.846 & 0.727 & 0.415 & 0.827 & 0.242 & 0.578 \\
NBA_1 & 0.370 & 0.513 & 0.487 & 0.196 & 0.833 & $\mathbf{0 . 0 1 4}^{*}$ & 0.718 \\
NBA & 0.375 & 0.300 & 0.337 & $\mathbf{0 . 0 2 2 ^ { * }}$ & 0.836 & 0.152 & 0.642 \\
NBA_All & 0.109 & 0.809 & 0.754 & 0.404 & 0.755 & 0.151 & 0.385 \\
BALWT_1 & 0.369 & 0.700 & 0.476 & 0.174 & 0.684 & $\mathbf{0 . 0 0 5} *^{* *}$ & 0.872 \\
BALWT & 0.544 & 0.414 & 0.311 & $\mathbf{0 . 0 2 9}$ & 0.807 & 0.144 & 0.739 \\
BALWT_All & 0.208 & 0.856 & 0.889 & 0.444 & 0.755 & 0.208 & 0.289 \\
P_All & 0.296 & 0.990 & 0.595 & 0.633 & 0.757 & 0.197 & 0.227 \\
\hline
\end{tabular}

${ }^{*} P<0.05,{ }^{* *} P<0.01$. SNP_2: rs81471381; SNP_3: rs80891106; SNP_4: rs81399474; SNP_5: rs81421148; SNP_6: rs81242222; SNP_7: rs81319839; SNP_8: rs81312912; SNP_9: rs80962240. TNB_1: total number born of the first parity; TNB: total number born of the second and subsequent parities; TNB_All: total number born of lifetime sow; NBA_1: total number born alive of the first parity; NBA: total number born alive of the second and subsequent parities; NBA_All: total number born alive of lifetime sow; BALWT_1: litter weight of piglets born alive of the first parity; BALWT: litter weight of piglets born alive of the second and subsequent parities; BALWT_All: litter weight of piglets born alive of lifetime sow; P_All: all parities of lifetime sow.

breeding work on the improvement of both LW and LN is mainly aimed at reproductive ability, each of these breeds has its own breed-specific characteristics, which are caused by differences in their genetic structure. In [15], BergfelderDrüing et al. revealed the genetic differentiation between LW and LN pigs. For GWAS, animals were divided into clusters by breeds, as well as interbreed clusters by the breeding farm. The results obtained on the basis of genotyping of the presented SNPs also showed the features of the frequencies and genotypes distribution associated with the pigs breed. To assess the effects of SNPs on litter traits, the animals under study were divided into two clusters according to the breed. Sell-Kubiak et al. [14] conducted GWAS for litter size in a LW pig population. According to their results, one of the significant SNPs associated with TNB was SNP rs80956812 (in our study, SNP_1) and SNP rs81471381 (in our study, SNP_2). In our studies, SNP_1 was monomorphic in LN. In LW, it has an additive effect on BALWT; effects on the other signs were not observed, possibly because of the low frequency of the SNP_1_AA genotype.

It is interesting to note that SNP_1 is localized in the intron of the small mothers against decapentaplegic 6 (SMAD6) gene (SSC1: 164,657,086-164,734,703; Sscrofa 11.1). The protein encoded by this gene belongs to the $S M A D$ family of proteins, which have been identified as signaling mediators of the transforming growth factor beta superfamily (TGF-beta). They are involved in a number of biological processes, including cell growth, morphogenesis, development, and immune responses [19]. SMAD1, SMAD2, $S M A D 3$, and SMAD5 are ligand-specific: SMAD1 and SMAD5 transform signals from bone morphogenetic proteins (BMPs); SMAD2 and SMAD3 mediate TGF-beta signaling, and SMAD4 acts as a common signaling component. $S M A D 6$ is completely different in structure from other $S M A D$ proteins; it forms stable associations with type I receptors and works as an inhibitor [20].The great majority of SMAD6 gene polymorphism studies are focused on identification of associations with human ovarian cancer $[21,22]$. Based on these studies results, we can assume that genetic variations of the SMAD6 gene can lead to changes in gene expression or regulation of the signaling function involved in the development of the reproductive process in pigs too.

Significant effects of SNP_2 on the analyzed traits were identified in LW and LN. It should be noted that these effects resulted from the heterozygous genotype (dominant effects). In LW, the effect of this SNP on BALWT only was determined, but in LN, effects were determined for all traits evaluated for the productive period (TNB_All, NBA_All, BALWT_All, P_ALL). SNP_2 is localized in the gene encoding hydroxymethylglutarate CoA-transferase succinate (SUGCT, SSC18: 53,639,593-54,283,251; Sscrofa 11.1). Sherman et al. [23] suggested that SUGCT (C7orf10) is a member of the coenzyme A family of class III transferases, based on a missense mutation (p.Arg336Trp) found in a homozygous state in several patients with type III glutaric aciduria. Further functional studies of catalytic activity and subcellular localization carried out by Marlaire et al. [24] confirmed that SUGCT (C7orf10) corresponds to succinylCoA: a mitochondrial enzyme glutarate CoA-transferase, involved in the metabolism of glutarate and possibly in the metabolism of longer dicarboxylic acids. Homologs of this enzyme are found in numerous bacterial operons, which also include the putative glutaryl CoA dehydrogenase, indicating that an enzyme with similar specificity exists in prokaryotes [24].

In studies of $\mathrm{He}$ et al. [16] on Chinese Erhualian pigs, 10 SNPs related to TNB and ovulation rate were presented. We have tested two of them, SNP rs80891106 (in our study, SNP_3) and SNP rs81399474 (in our study, SNP_4). Significant effects of SNP_3 on litter traits of first parity and on P_All were identified in LW. Possible functional features of SNP_3 are unknown yet, because of its localization in the noncoding region, and the nearby genes RF00001 (73,234,200-73,234,325; Sscrofa 11.1) and ENSSSCGG000032058 (73,861,910-73,911,853; Sscrofa 11.1) encode a ribosomal RNA and long noncoding RNA, respectively. However, data obtained by He et al. [16] and our results indicate the connection between SNP_3 and litter traits of pigs, whose mechanisms are still difficult to explain.

According to the LW pig research conducted by Wang et al. [17], we have chosen SNP rs81319839 (in our study, SNP_7), SNP rs81312912 (in our study, SNP_8), and SNP 
rs80962240 (in our study, SNP_9). In our work, the effects of SNP_7 on TNB_1 in LW and on NBA_1 and BALWT_1 in LN were established. This shows the significance of SNP_7 for the productivity of sows during the first farrowing period. In general, the first litter in the sows' life is associated with great stress for the body; productivity is realized on the background of the immature endocrine system of growing young animals [25]. In this regard, it is interesting to note that SNP_7 is localized in the SSC4 intergenic region, but one of the nearby genes is MTBP (SSC4: 18,535,841-18,609,163; Sscrofa 11.1). Brady et al. [26] showed the significant role of the MTBP gene (MDM2 binding protein) in the mechanisms ensuring the destruction of the $\mathrm{p} 53$ protein by the $M D M 2$ ubiquitinated ligase. The $p 53$ protein is a critical coordinator of a wide range of stress reactions in the body. The $p 53$ gene is expressed with constant activity, but the protein has a very short period of life, regulated by E3 ubiquitinated ligases. The most studied and probably significant of them is the ubiquitinated ligase $M D M 2$. The $p 53$ regulation is provided by the negative feedback implemented by MDM2 using MTBP. It is very important for the organism to regulate p53 activity firmly and accurately to prevent inappropriate activation leading to the death of cell and possibly the death of organism [26]. This requires strict regulation of the correct balance between p53 and MDM2. Expression of MDM2 is observed in a wide range of values and varies in different tissues, but the highest level is found in the testes and ovaries [27]. Interestingly, $M T B P$ mRNA also has the highest level in these tissues [28]. In general, the role of MTBP in inhibiting the cis- and stimulating the trans-reactions of ubiquitin ligase E3 MDM2 suggests that $M T B P$ can also provide the functions which are necessary to regulate the reproductive activity of sows, especially during the first parity.

Among the studied SNPs, the most significant effect on variability of studied traits in the investigated population has been obtained for SNP_5 (SNP rs81421148). This result was described by Bergfelder-Drüing et al. [15] in their research on GWAS associated with the number of piglets born alive in LW sows. Our results have demonstrated significant association of SNP_5 with TNB, NBA, and BALWT in LW and LN. SNP_5 is localized in AKT3 gene (SSC10: 16,441,465-16,741,745). This gene codes serine/threonine protein kinase. Three subtypes of protein kinases, AKT1, AKT2, and AKT3, have been identified in mammals; they play a key role in metabolism of glucose and angiogenesis as well as in PI3K/AKT signaling pathway, which regulates cell cycle and apoptosis. Despite the differences between the specific functions of $A K T$ isoforms, all 3 subtypes are potential candidate genes associated with phenotype variation in humans and animals. The single-nucleotide polymorphism of AKT3 (rs4590656) was found to be associated with three physiological parameters (hemoglobin, hematocrit, and red blood cell count) in people with chronic altitude sickness, indicating a strong association of this gene with angiogenesis [29]. A study by Gottlob et al. [30] showed that the level of $A T P$ in the fibroblasts cell line in mice significantly depends on the AKT family. Bionaz and Loor [31] determined that the $A K T 1$ and $A K T 3$ levels of expression increase considerably during lactation periods in cattle. Wang et al. [32] revealed the effect of the AKT3 gene polymorphism on the early maturity in rabbits. Chen et al. [33] demonstrated the gene polymorphism association with the components of muscle tissue in broilers. Liu et al. [34] found three insertions in AKT3 gene associated with Xiang pig fattening traits.

The AKT3 functional role is realized through the PI3K/ AKT pathway. In mammals, an inhibition of the PI3K/AKT pathway blocks almost all insulin metabolic actions, including stimulation of glucose transport [35]. It is estimated that the $P I 3 K / A K T$ pathway is involved in the regulation of folliculogenesis and oogenesis. The role of $A K T$ in mammalian ovaries was evaluated by gene knockout. Female mice with $A K T 1$ deficiency have demonstrated that the fertility decreases, the estrus delays for about 5 days, the age of the first litter increases, and the average litter size decreases. Primary follicles oocytes in females with $A K T 1$ deficiency are larger than those in wild-type animals, and sometimes the follicles contain several oocytes. The effects of AKT2 and AKT3 on fertility are still unclear. The AKT controls the activity of some transcription factors of the forkhead box protein O1 or FOXO family (FOXO1, FOXO3, and FOXO4). The transcription factors FOXO1, FOXO3, and FOXO4 are also involved in the control of folliculogenesis [36]. On the basis of all the above, it can be assumed that AKT3 is a perspective gene candidate for reproductive traits of pigs.

\section{Conclusion}

The results obtained showed the pleiotropic effect of the selected SNPs on the traits related to the litter size, the litter weight, and the amount of parities. Here, we demonstrated the local genotyping systems of some SNPs from PigQTLdb, which can be used in further studies on the genetic architecture and its connection with the pig productivity traits. The analysis of the SNPs alleles and genotypes frequency distribution revealed features associated with the pigs' breed, which must be considered when evaluating the effects of SNPs. The results showed significant effects of SNPs on litter traits in LW and LN. AKT3 was identified as a promising candidate gene, whose biological functions, as well as the results obtained in our work and in other studies, indicate its potential role in the reproductive process regulation in pigs. In general, the data obtained help to explain the genetic mechanisms of reproductive traits.

\section{Abbreviations}

QTL: Quantitative trait loci

GWAS: Genome-wide association study

SNP: $\quad$ Single-nucleotide polymorphism

TNB: $\quad$ Total number born

NBA: $\quad$ Total number born alive

PigQTLdb: Pig quantitative trait loci database

PCA: $\quad$ Principal component analysis

LW: $\quad$ Large White

LN: Landrace

AKT3: $\quad$ AKT serine/threonine kinase 3 gene 
BLUP: $\quad$ Best linear unbiased prediction

NCBI: National Center for Biotechnology Information

PCR-RFLP: Polymerase chain reaction-restriction fragment length polymorphism

PSQ: $\quad$ Pyrosequencing method

BALWT: $\quad$ Litter weight of piglets born alive

TNB_1: $\quad$ Total number born of the first parity

NBA_1: Total number born alive of the first parity

BALWT_1: Litter weight of piglets born alive of the first parity

TNB_All: Total number born of the lifetime sow

NBA_All: Total number born alive of the lifetime sow

BALWT_All: Litter weight of piglets born alive of the lifetime sow

P_All: $\quad$ All parities of the lifetime sow

SMAD: Small mothers against decapentaplegic

SSC: $\quad$ Sus scrofa chromosome.

\section{Data Availability}

The datasets produced and/or analyzed during the current study are available from the corresponding author on reasonable request.

\section{Additional Points}

Highlights. The effect on litter traits in LW was determined for SNPs rs80956812, rs81471381, rs81421148, and rs81399474. The effect on litter traits in LN was determined for SNPs rs 81421148 and rs81319839. The AKT3 gene can be considered as a potential candidate gene. Local genotyping systems of some SNPs from PigQTLdb can be used in further studies.

\section{Conflicts of Interest}

The authors declare that they have no conflicts of interest.

\section{Acknowledgments}

The reported study was funded by RFBR according to the research project no. 18-016-00050-A.

\section{Supplementary Materials}

Add. File 1: the names of the SNPs in accordance with the chips for genotyping on the basis of Ensembl Release 96 (April 2019). Add. File 2: Table F2_1-the results of additive and dominant effects of SNPs in LW; Table F2_2-the results of additive and dominant effects of SNPs in LN. (Supplementary Materials)

\section{References}

[1] P. M. Visscher, N. R. Wray, Q. Zhang et al., "10 Years of GWAS discovery: biology, function, and translation," The American Journal of Human Genetics, vol. 101, no. 1, pp. 5-22, 2017.
[2] H. Zhang, Z. Wang, S. Wang, and H. Li, "Progress of genome wide association study in domestic animals," Journal of Animal Science and Biotechnology, vol. 3, no. 1, p. 26, 2012.

[3] Z. Jiang, H. Wang, J. J. Michal et al., "Genome wide sampling sequencing for SNP genotyping: methods, challenges and future development," International Journal of Biological Sciences, vol. 12, no. 1, pp. 100-108, 2016.

[4] H. Aliloo, J. Pryce, O. González-Recio, B. G. Cocks, and B. J. Hayes, "Accounting for dominance to improve genomic evaluations of dairy cows for fertility and milk production traits," Genetics Selection Evolution, vol. 48, no. 1, p. 8, 2016.

[5] Y. Guo, Y. Huang, L. Hou et al., "Genome-wide detection of genetic markers associated with growth and fatness in four pig populations using four approaches Genetics," Selection Evolution, vol. 49, no. 1, p. 21, 2017.

[6] E. F. Knol, B. Nielsen, and P. W. Knap, "Genomic selection in commercial pig breeding," Animal Frontiers, vol. 6, no. 1, pp. 15-22, 2016.

[7] P. Sun, Q. Yang, K. Wang et al., "Single step genome-wide association studies based on genotyping by sequence data reveals novel loci for the litter traits of domestic pigs," Genomics, vol. 110, no. 3, pp. 171-179, 2018.

[8] J. Häggman and P. Uimari, "Novel harmful recessive haplotypes for reproductive traits in pigs," Journal of Animal Breeding and Genetics, vol. 134, no. 2, pp. 129-135, 2017.

[9] T. Zhang, L.-G. Wang, H.-B. Shi et al., "Hritabilities and genetic and phenotypic correlations of litter uniformity and litter size in Large White sows," Journal of Integrative Agriculture, vol. 15, no. 4, pp. 848-854, 2016.

[10] A. Crovetti, R. Bozzi, C. Pugliese, and A. Acciaioli, "Genetic parameters of productive and reproductive traits in Cinta Senese pig," Italian Journal of Animal Science, vol. 4, no. 2, pp. $82-84,2010$.

[11] J.-H. Lee, K.-D. Song, H.-K. Lee, K.-H. Cho, H.-C. Park, and K.-D. Park, "Genetic parameters of reproductive and meat quality traits in Korean berkshire pigs," Asian-Australasian Journal of Animal Sciences, vol. 28, no. 10, pp. 1388-1393, 2015.

[12] J. Ye, C. Tan, X. Hu, A. Wang, and Z. Wu, "Genetic parameters for reproductive traits at different parities in large white pigs," Journal of Animal Science, vol. 96, no. 4, pp. 1215-1220, 2018.

[13] S. Bakoev, L. V. Getmantseva, F. S. Bakoev et al., "Survey of SNP associated with total number born and total number born alive in pig," Genes (Basel), vol. 11, no. 5, p. E491, 2020.

[14] E. Sell-Kubiak, N. Duijvesteijn, M. S. Lopes et al., "Genomewide association study reveals novel loci for litter size and its variability in a Large White pig population," BMC Genomics, vol. 16, p. 1049, 2015.

[15] S. Bergfelder-Drüing, C. Grosse-Brinkhaus, B. Lind et al., "A genome-wide association study in large white and Landrace pig populations for number piglets born alive," PLoS One, vol. 10, no. 3, 2015.

[16] L. C. He, P. H. Li, X. Ma et al., "Identification of new single nucleotide polymorphisms affecting total number born and candidate genes related to ovulation rate in Chinese Erhualian pigs," Animal Genetics, vol. 48, no. 1, pp. 48-54, 2016.

[17] Y. Wang, X. Ding, Z. Tan et al., "Genome-wide association study for reproductive traits in a Large White pig population," Animal Genetics, vol. 49, no. 2, pp. 127-131, 2018.

[18] D. S. Falconer and T. F. C. Mackay, Introduction to Quantitative Genetics, Addison Wesley Longman, Harlow, UK, 4th edition, 1996. 
[19] J. Yin, K. Lu, J. Lin et al., "Genetic variants in TGF- $\beta$ pathway are associated with ovarian cancer risk," PLoS One, vol. 6, no. 9, Article ID e25559, 2011.

[20] H. L. Tan, E. Glen, A. Töpf et al., "Nonsynonymous variants in theSMAD6gene predispose to congenital cardiovascular malformation," Human Mutation, vol. 33, no. 4, pp. 720-727, 2012.

[21] N. J. Bowen, L. D. Walker, L. V. Matyunina et al., "Gene expression profiling supports the hypothesis that human ovarian surface epithelia are multipotent and capable of serving as ovarian cancer initiating cells," BMC Medical Genomics, vol. 2, p. 71, 2009.

[22] C. Le Page, M. L. Puiffe, L. Meunier et al., "BMP-2 signaling in ovarian cancer and its association with poor prognosis," Journal Ovarian Research, vol. 2, no. 1, 2009.

[23] E. A. Sherman, K. A. Strauss, S. Tortorelli et al., "Genetic mapping of glutaric aciduria, type 3, to chromosome 7 and identification of mutations in C7orf10," The American Journal of Human Genetics, vol. 83, no. 5, pp. 604-609, 2008.

[24] S. Marlaire, E. Van Schaftingen, and M. Veiga-da-Cunha, "C7orf10 encodes succinate-hydroxymethylglutarate CoAtransferase, the enzyme that converts glutarate to glutarylCoA," Journal of Inherited Metabolic Disease, vol. 37, no. 1, pp. 13-19, 2014.

[25] Y. Koketsu, S. Tani, and R. Iida, "Factors for improving reproductive performance of sows and herd productivity in commercial breeding herds," Porcine Health Management, vol. 3, no. 1, p. 1, 2017.

[26] M. Brady, N. Vlatkovic, and M. T. Boyd, "Regulation of p53 and MDM2 activity by MTBP," Molecular and Cellular Biology, vol. 25, no. 2, pp. 545-553, 2005.

[27] S. S. Fakharzadeh, S. P. Trusko, and D. L. George, "Tumorigenic potential associated with enhanced expression of a gene that is amplified in a mouse tumor cell line," The EMBO Journal, vol. 10, no. 6, pp. 1565-1569, 1991.

[28] M. T. Boyd, N. Vlatkovic, and D. S. Haines, "A novel cellular protein (MTBP) binds to MDM2 and induces a G1Arrest that is suppressed by MDM2," Journal of Biological Chemistry, vol. 275, no. 41, pp. 31883-31890, 2000.

[29] N. E. Buroker, "Regulatory SNPs and transcriptional factor binding sites in ADRBK1, AKT3, ATF3, DIO2, TBXA2R and VEGFA," Transcription, vol. 5, no. 4, Article ID e964559, 2014.

[30] K. Gottlob, N. Majewski, S. Kennedy, E. Kandel, R. B. Robey, and N. Hay, "Inhibition of early apoptotic events by Akt/PKB is dependent on the first committed step of glycolysis and mitochondrial hexokinase," Genes \& Development, vol. 15, no. 11, pp. 1406-1418, 2001.

[31] M. Bionaz and J. J. Loor, "Gene networks driving bovine mammary protein synthesis during the lactation cycle," Bioinformatics Biology Insights, vol. 5, pp. 83-98, 2011.

[32] L. Wang, X. Jia, S. Chen, J. Wang, and S. Lai, "Polymorphisms of PIK3CA and AKT3 genes and their association with growth traits of rabbits," Journal of Sichuan Agricultural University, vol. 34, no. 3, pp. 374-380, 2016.

[33] S. Chen, J. An, L. Lian et al., "Polymorphisms in AKT3, FIGF, PRKAG3, and TGF- $\beta$ genes are associated with myofiber characteristics in chickens," Poultry Science, vol. 92, no. 2, pp. 325-330, 2013.

[34] C. Liu, X. Ran, J. Wang, S. Li, and J. Liu, "Detection of genomic structural variations in Guizhou indigenous pigs and the comparison with other breeds," PLoS One, vol. 13, no. 3, 2018.

[35] B. Cheatham, C. J. Vlahos, L. Cheatham, L. Wang, J. Blenis, and C. R. Kahn, "Phosphatidylinositol 3-kinase activation is required for insulin stimulation of pp70 S6 kinase, DNA synthesis, and glucose transporter translocation," Molecular and Cellular Biology, vol. 14, no. 7, pp. 4902-4911, 1994.

[36] M. Shen, Z. Liu, B. Li et al., "Involvement of FoxO1 in the effects of follicle-stimulating hormone on inhibition of apoptosis in mouse granulosa cells," Cell Death \& Disease, vol. 5, no. 10, p. e1475, 2014. 